\title{
CD47-retargeted oncolytic adenovirus armed with melanoma differentiation-associated gene-7/interleukin-24 suppresses in vivo leukemia cell growth
}

\author{
Gongchu Li ${ }^{1}$, Hu Wu ${ }^{1}$, Lianzhen Cui ${ }^{1}$, Yajun Gao ${ }^{1}$, Lei Chen ${ }^{1}$, Xin Li ${ }^{1}$, Tianxiang Liang ${ }^{1}$, \\ Xinyan Yang ${ }^{1}$, Jianhong Cheng ${ }^{1}$, Jingjing Luo ${ }^{1}$ \\ ${ }^{1}$ College of life sciences, Zhejiang Sci-Tech University, Hangzhou, Zhejiang, China \\ Correspondence to: Gongchu Li, e-mail: Igc@zstu.edu.cn \\ Keywords: oncolytic adenovirus, CD47, thrombospondin-1, leukemia, interleukin-24 \\ Received: May 07, $2015 \quad$ Accepted: October 23, $2015 \quad$ Published: November 02, 2015
}

\begin{abstract}
Our previous studies have suggested that harboring a soluble coxsackieadenovirus receptor-ligand (sCAR-ligand) fusion protein expression cassette in the viral genome may provide a universal method to redirect oncolytic adenoviruses to various membrane receptors on cancer cells resisting to serotype 5 adenovirus infection. We report here a novel oncolytic adenovirus vector redirected to CD47+ leukemia cells though carrying a SCAR-4N1 expression cassette in the viral genome, forming Ad.4N1, in which $4 \mathrm{N1}$ represents the C-terminal CD47-binding domain of thrombospondin-1. The infection and cytotoxicity of Ad.4N1 in leukemia cells were determined to be mediated by the 4N1-CD47 interaction. Ad.4N1 was further engineered to harbor a gene encoding melanoma differentiation-associated gene-7/ interleukin-24 (mda-7/IL-24), forming Ad.4N1-IL24, which replicated dramatically faster than Ad.4N1, and elicited significantly enhanced antileukemia effect in vitro and in a HL60/Luc xenograft mouse model. Our data suggest that Ad.4N1 could act as a novel oncolytic adenovirus vector for CD47+ leukemia targeting gene transfer, and Ad.4N1 harboring anticancer genes may provide novel antileukemia agents.
\end{abstract}

\section{INTRODUCTION}

CD47, or integrin associated protein (IAP), is a transmembrane protein which serves as a receptor for thrombospondin (TSP) family members, as well as a ligand for macrophage signal-regulatory protein $\alpha(\operatorname{SIRP} \alpha)[1] . \mathrm{CD} 47$ has a higher level of expression on acute myeloid leukemia blasts and leukemia stem cells, liver cancer stem cells, as well as pancreatic stem cells, as compared with their normal counterparts [2-4]. The CD47-SIRP $\alpha$ interaction between acute myeloid leukemia cells and macrophages inhibits the phagocytosis by macrophages [5]. Therapeutic antibodies against CD47 selectively eliminated acute myeloid leukemia [6], lymphoblastic leukemia [7], pancreatic cancer stem cells [3], breast cancer [8], and hepatocellular carcinoma [4] through inducing the phagocytosis of cancer cells, directly inducing apoptosis, or sensitizing cancer cells to chemotherapy. In addition, an engineered high affinity $\operatorname{SIRP} \alpha$ variant elicited dramatic synergistic effect with various tumor specific monoclonal antibodies through inducing the phagocytosis of cancer cells by macrophages [9]. Furthermore, delivery of anti-CD47 siRNA by nanoparticles inhibited melanoma tumor growth and lung metastasis [10]. CD47 blockade on cancer cells not only induced macrophage phagocytosis, but also activated antitumor CD8+ cytotoxic T cell response [11]. Collectively, CD47 provides a promising target for cancer therapies and have attracted wide interests.

Due to lytic replication, efficient gene transfer, and low pathogenicity, oncolytic adenovirus, or conditionally replicating adenoviruses, has become a promising strategy for cancer therapy [12-15]. In this strategy, adenoviruses are engineered to selectively replicate and induce cytotoxicity in cancer cells. The oncolytic modifications include the deletion of viral genes which are essential to complete the viral lytic cycle in normal cells, but not in tumor cells, or controlling the expression of genes regulating viral replication with tumor-specific promoters [16]. Coxsackie-adenovirus recptor (CAR) is the primary receptor for the infection of serotype 5 (Ad5) adenoviruses, the most commonly 
used adenoviral vector in cancer gene therapy $[17,18]$. However, leukemia cells only express low levels of CAR, which results in resistance to Ad5 infection [19]. To redirect Ad5 to leukemia cells, adenoviruses were modified through genetically incorporating receptorspecific ligand peptides into the viral fibers, or altering viral tropism through the replacement of the fiber knob alone or together with the shaft domain to form chimeric fibers [20-23]. Recombinant adaptor proteins containing CAR and specific ligands were also utilized for bridging Ad5 to various cell membrane receptors [24, 25].

Previously, we designed a novel strategy to redirect oncolytic adenoviruses to leukemia cell membrane receptors though carrying a sCAR-ligand expression cassette in the viral genome [26]. For example, to retarget oncolytic adenoviruses to interleukin-3 receptor $\alpha$ subunit (CD123), a sCAR-IL3 expression cassette was genetically inserted into the viral genome. During viral packaging, the sCAR-IL3 fusion protein would be expressed in packaging cells and noncovalently installed on viral surface, which bridged oncolytic adenoviruses to CD123+ leukemia cells. After infection and replication in leukemia cells, the sCAR-IL3 expression would help newly produced oncolytic adenoviruses to be further modified and infect more leukemia cells. Therefore, harboring sCAR-ligand expression cassette in the viral genome may become a universal method to redirect oncolytic adenoviruses to various membrane receptors on cancer cells resisting to Ad5 adenovirus infection.

In addition to therapeutic antibodies described above, oncolytic adenoviruses may provide an alternative therapeutic method for targeting CD47+ leukemia. In the work presented, we constructed a novel CD47 targeting oncolytic adenovirus through genetically modifying Ad.sp-E1A, a previously reported conditionally replicative oncolytic adenovirus in which the viral E1A is controlled by a survivin promoter [27], to carry a sCAR-4N1 expression cassette in the viral genome, forming Ad.4N1. Peptide 4N1 is the C-terminal CD47/IAP-binding domain of TSP-1 with the amino acid sequence RFYVVMWK [28]. Moreover, Ad.4N1 was further armed with a gene encoding melanoma differentiation-associated gene-7/ interleukin-24 (mda-7/IL-24), a well known anticancer agent described previously [27, 29, 30], to form oncolytic adenovirus Ad.4N1-IL24. The in vitro and in vivo therapeutic effects of Ad.4N1 and Ad.4N1-IL24 against CD47+ leukemia cells were evaluated.

\section{RESULTS}

\section{In vitro characterization of $\mathrm{SCAR}-4 \mathrm{~N} 1$ fusion protein}

Recombinant sCAR-4N1 protein was designed to contain a 6his-tag, a human coxsackie-adenovirus receptor extracellular domain ( $\mathrm{SCAR}$ ), a short flexible linker, and a TSP-1 C-terminal 4N1 peptide (Figure 1A). The expression and purification of $\mathrm{sCAR}-4 \mathrm{~N} 1$ from a bacterial expression system were examined by SDS-PAGE followed by Coomassie Brilliant Blue staining. As shown in Figure 1B, a relatively pure protein with expected molecular weight was obtained. To test the activity of sCAR-4N1 fusion proteins, CD47+ leukemia cell line K562 was treated with sCAR-4N1 followed by Hoechst 33342 staining. PBS was used as the control. As compared to the control, sCAR-4N1 treatment dramatically induced apoptosis in K562 cells (Figure 1C). Furthermore, K562 cells were treated with Ad-EGFP, a replication-defective adenovirus expressing enhanced green fluorescent protein, combined with sCAR-4N1. K562 cells treated with Ad-EGFP alone served as the control. As determined by fluorescent microscopy (Figure 1D), sCAR-4N1 significantly increased the Ad-EGFP infection in K562 cells. Therefore, our results determined that SCAR-4N1 fusion protein could not only induce apoptosis, but also facilitate adenoviral infection in K562 cells.

\section{Oncolytic adenoviruse carrying sCAR-4N1 expression cassette elicited cytotoxicity to CD47+ leukemia cells}

We further engineered a previously reported oncolytic adenovirus Ad.sp-E1A to harbor a cytomegalovirus (CMV) promoter controlled sCAR-4N1 expression cassette, forming a novel oncolytic adenovirus Ad.4N1 (Figure 2A). To evaluate the antiproliferative effect of Ad.4N1, CD47 and survivin-positive leukemia cells K562 [31, 32] and HL60 $[33,34]$ were treated with Ad.sp-E1A or Ad.4N1. PBS was used as the control. As shown in Figure $2 \mathrm{~B}$ and $2 \mathrm{C}$, compared to Ad.sp-E1A, Ad.4N1 significantly suppressed the in vitro proliferation of both K562 and HL60 cells, at dose- and time-dependent manners. Therefore, data demonstrated that Ad.4N1 successfully infected and induced antiproliferative effect on CD47+ leukemia cells. To further analyze the underlying mechanism of cytotoxicity induced by Ad.4N1, HL60 cells treated with PBS, Ad.sp-E1A, or Ad.4N1 were investigated for apoptotic signaling elements through Western blot. As shown in Figure 2D, Ad.4N1 dramatically induced the upregulation of proapoptotic factor Bax. Interestingly, Ad.4N1 also slightly upregulated the levels of antiapoptotic factor B-cell lymphoma 2 (Bcl-2), but without significant effect on the cleavage of caspase 3 . Our data suggest that Ad.4N1 may induce antiproliferative effect on HL60 cells through upregulating Bax, and the upregulation of Bcl-2 may counteract the cytotoxic effect of Ad.4N1.

\section{Ad.4N1 suppressed leukemia cell proliferation through 4N1-CD47 interaction}

To determine that Ad.4N1 infected leukemia cells through CD47, a recombinant human CD47 Fc chimera 
(rhCD47-Fc) was combined with Ad.4N1 to treat K562 cells, followed by MTT assay for cell viability. As shown in Figure 3A, rhCD47-Fc significantly counteracted with the Ad.4N1 induced proliferation inhibition at a dosedependant manner, indicating that Ad.4N1 used CD47 as the cell membrane receptor for viral internalization. Furthermore, the antiproliferative effect of Ad.4N1 on HL60 was compared to Ad.IL3, a previously produced oncolytic adenovirus expressing sCAR-IL3 fusion proteins [26]. Results showed that Ad.4N1, but not Ad.IL3, timedependently suppressed the proliferation of HL60 (Figure
3B). Taken together, our data demonstrated that Ad.4N1 infected and suppressed leukemia cell proliferation through the 4N1-CD47 interaction.

\section{Ad.4N1 armed with IL-24 elicited higher cytotoxicity to leukemia cells in vitro and in vivo}

To further elevate the antiproliferative effect of Ad.4N1, viral E1B was deleted, and a resulted restriction site was used to harbor a CMV promoter controlled human IL-24 expression cassette, forming Ad.4N1-IL24.
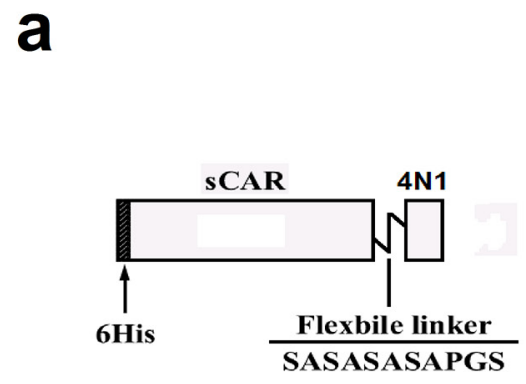

b

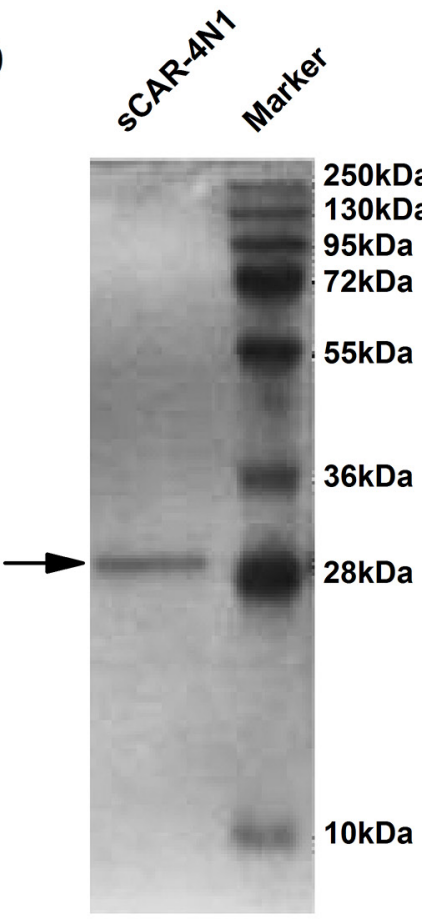

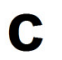
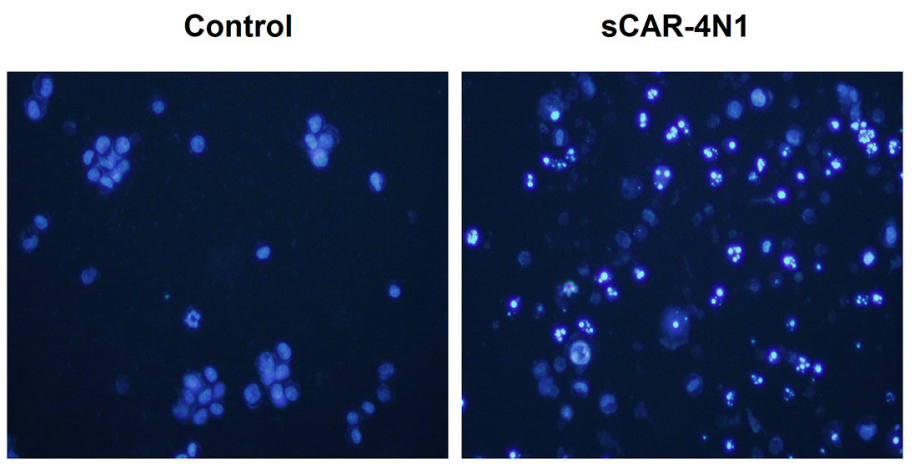

d
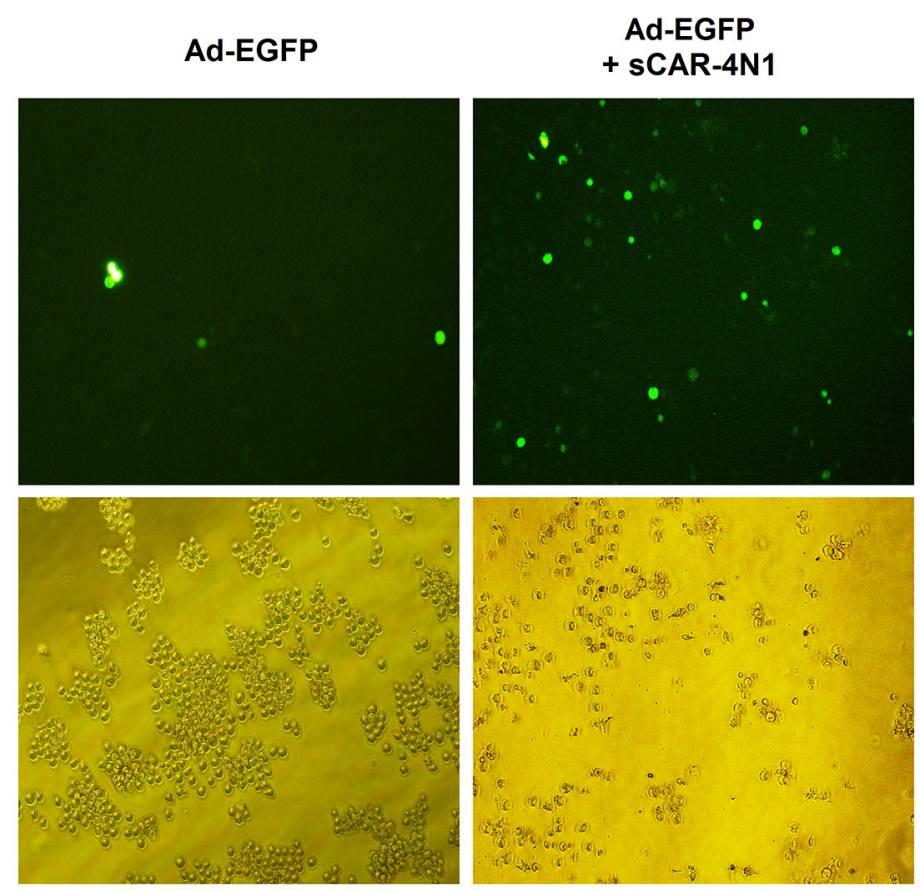

Figure 1: The in vitro characterization of sCAR-4N1 fusion protein. a. Schematic structure of the sCAR-4N1 fusion proteins. The recombinant protein consists of a 6his-tag, an extracellular domain of CAR with 239 amino acids, a flexible linker (SASASASAPGS), and the 4N1 peptide. b. The bacterial production of recombinant sCAR-4N1. The sCAR-4N1 was purified through a Ni-NTA-Sepharose column, and subjected to SDS-PAGE followed by Coomassie Brilliant Blue staining. c. The cytotoxicity of recombinant sCAR-4N1 to K562 cells. K562 was treated with $20 \mu \mathrm{g} / \mathrm{ml}$ of sCAR-4N1 followed by Hoechst 33342 staining. PBS was used as the control. Nuclear staining and apoptosis were observed under a fluorescent microscope. Shown is a representative experiment from 3 separate repeats. d. K562 cells at $2.5 \times 10^{5}$ cells per well were treated with $4 \times 10^{8}$ viral particles (vp) of Ad-EGFP pre-mixed with $10 \mu \mathrm{g}$ of sCAR-4N1. Cells treated with Ad-EGFP alone served as the control. After 2 days, GFP-positive cells were analyzed under a fluorescence microscope. Shown is a representative experiment from 3 separate repeats. 


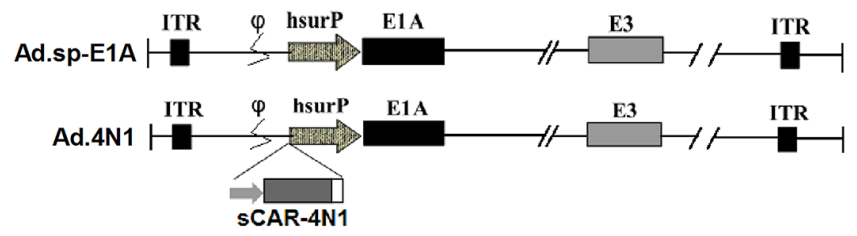

b $\quad$ K562
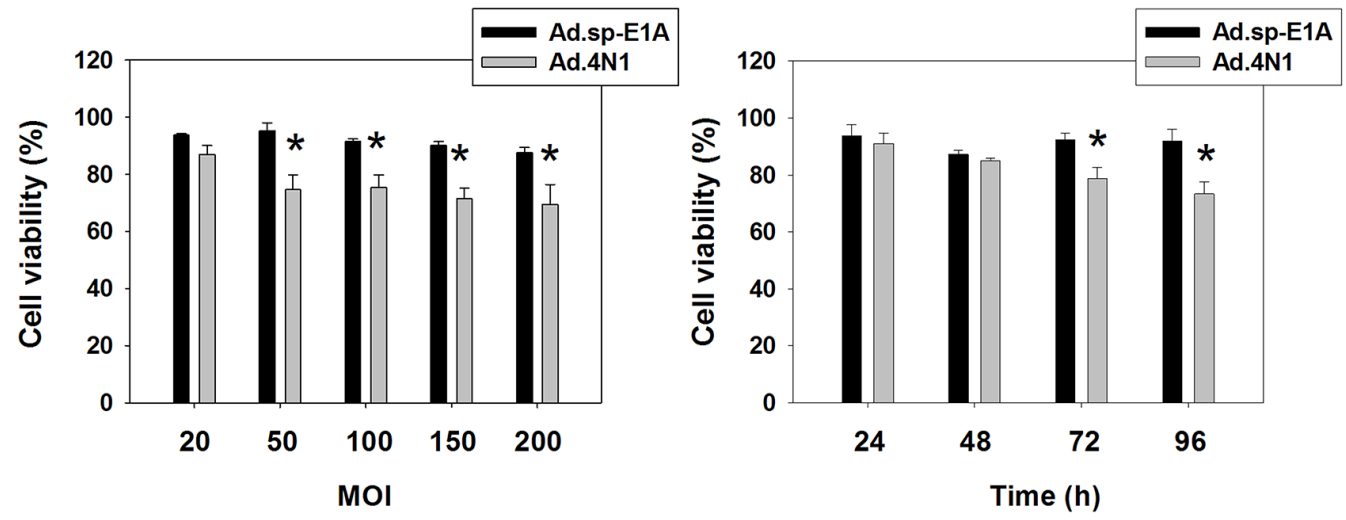

C HL60
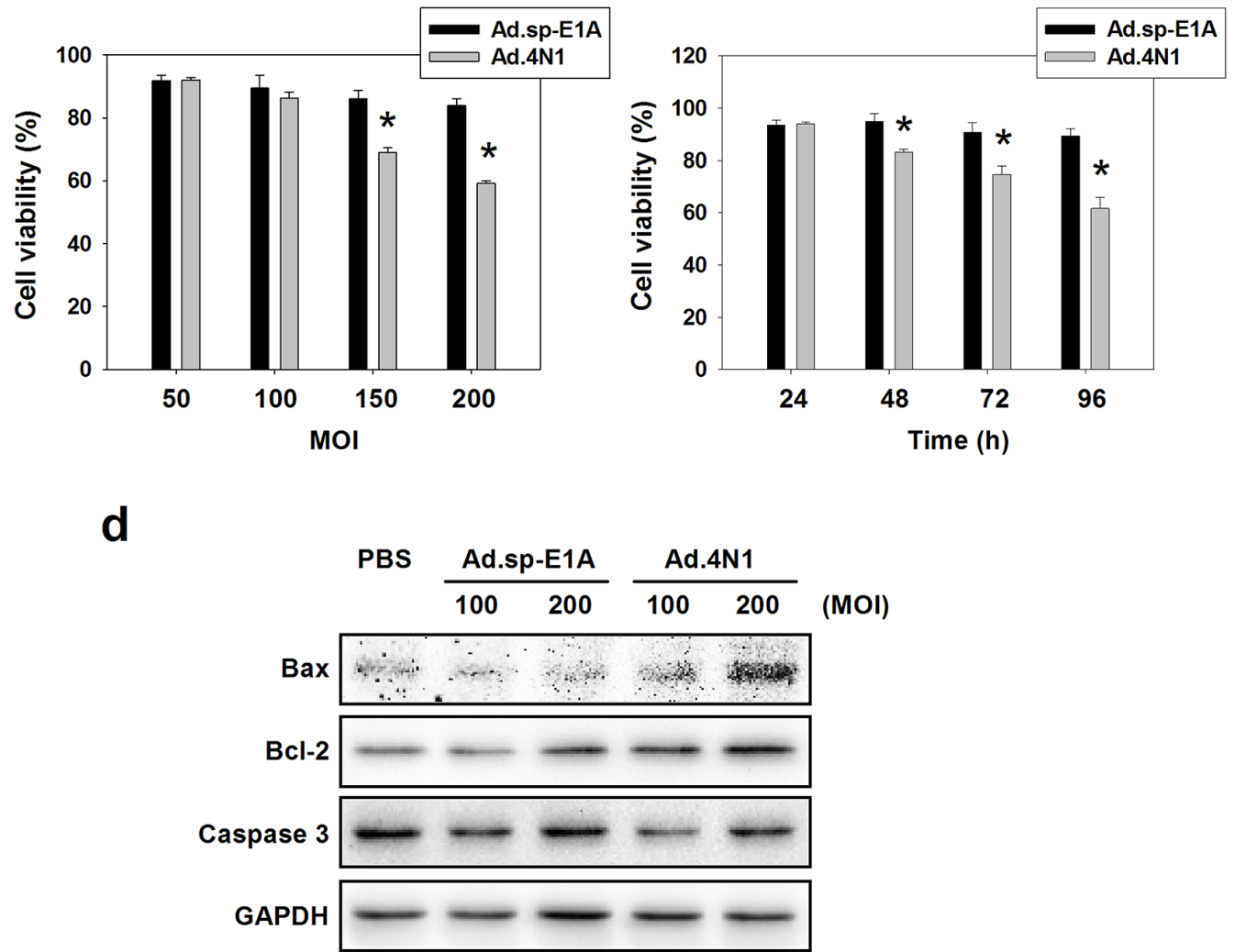

Figure 2: In vitro characterization of oncolytic adenovirus Ad.4N1. a. Schematic structure of Ad.sp-E1A and Ad.4N1. As shown in Ad.sp-E1A and Ad.4N1, the viral E1A promoter was replaced by a human survivin promoter (hsurP). Ad.4N1 contains a sCAR-4N1 expression cassette. ITR: inverted terminal repeat; hsurP: human survivin promoter. b. K562 or c. HL60 cells were treated with Ad.spE1A or Ad.4N1 at MOIs indicated for $72 \mathrm{~h}$ (left chart), or treated with Ad.sp-E1A or Ad.4N1 at 150 MOI for the time period indicated (right chart). Cell viability was analyzed by MTT assay. Values were calculated as percent of PBS control and presented as mean $\pm \mathrm{SEM}$. $\left({ }^{*} p<0.05\right)$ d. HL60 cells treated with PBS, Ad.sp-E1A or Ad.4N1 for $48 \mathrm{~h}$ were analyzed by Western blot for levels of Bax, Bcl-2, and Caspase 3. GAPDH served as the loading control. 
a

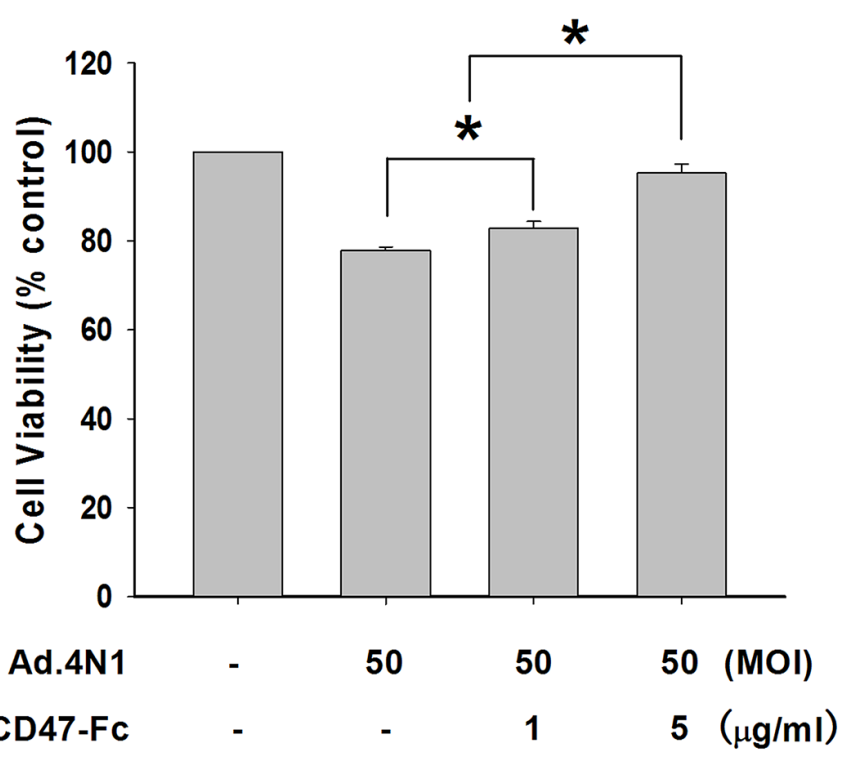

b
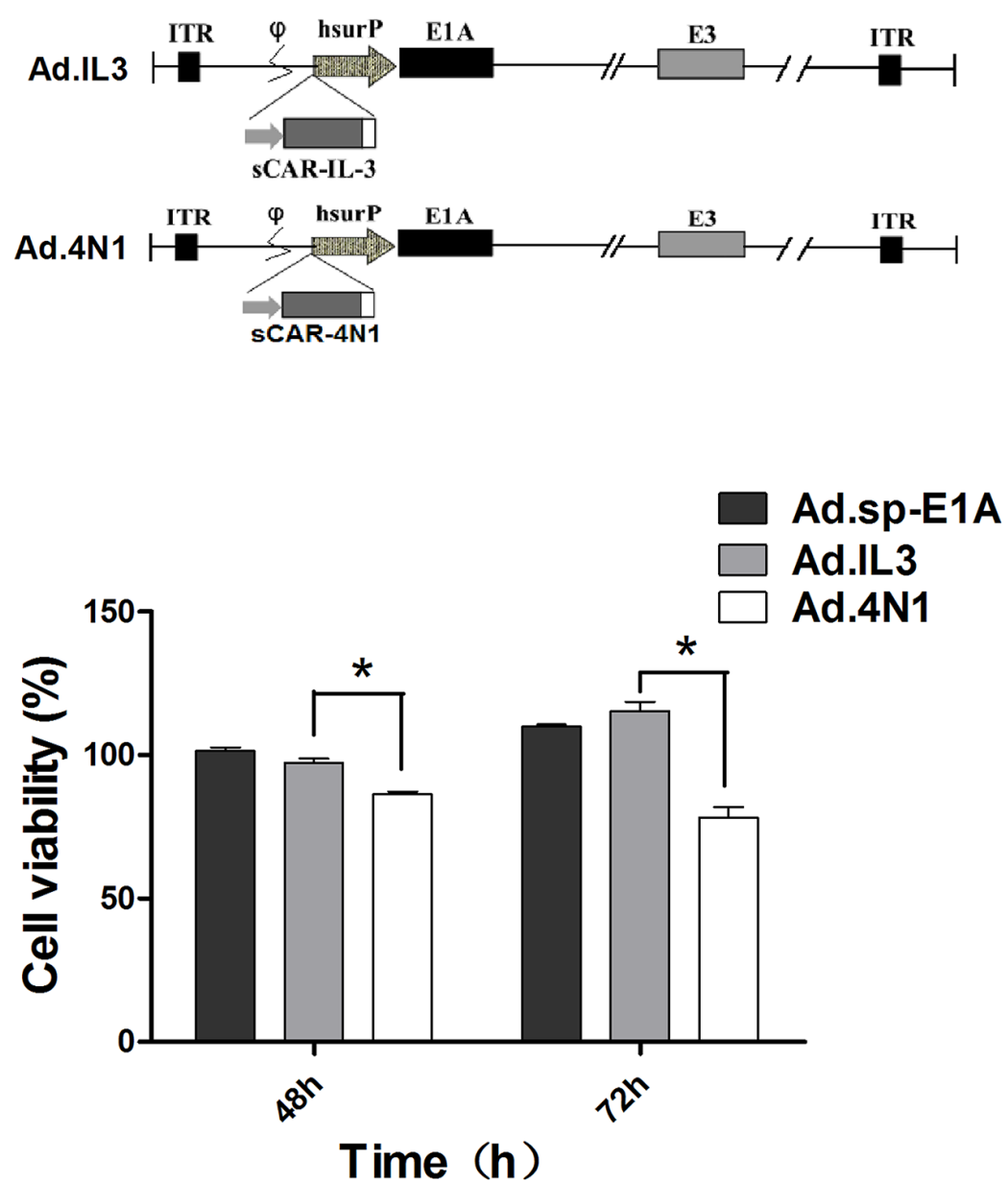

Figure 3: Ad.4N1 suppressed leukemia cell proliferation through the 4N1-CD47 interaction. a. K562 cells were treated with Ad.4N1 combined with recombinant human CD47 Fc chimera (rhCD47-Fc) at dosages indicated. Cell viability was then determined by MTT assay after $96 \mathrm{~h}$. Values were calculated as percent of PBS control and presented as mean \pm SEM. b. HL60 cells were treated with Ad.sp-E1 A, Ad.IL3, or Ad.4N1 at 200 MOI for the time period indicated. Cell viability was analyzed by MTT assay. Values were calculated as percent of PBS control and presented as mean \pm SEM. $\left({ }^{*} p<0.05\right)$ 
The schematic structure of the viral genome of Ad.4N1IL24 was shown in Figure 4A. To evaluate the cytotoxic effect of Ad.4N1-IL24, K562 and HL60 cells were treated with Ad.4N1 or Ad.4N1-IL24 at MOIs indicated, followed by MTT assay. PBS served as the control. As shown in Figure 4B and 4C, Ad.4N1-IL24 suppressed the in vitro proliferation of K562 and HL60 at a significantly higher level than Ad.4N1. We then performed further tests on the safety of Ad.4N1-IL24. Because normal human blood cells were unavailable in our studies, lung cancer cell line A549 and normal lung cell line BEASE-2B were analyzed and compared. As shown in Supplementary Figure S1,

\section{a}

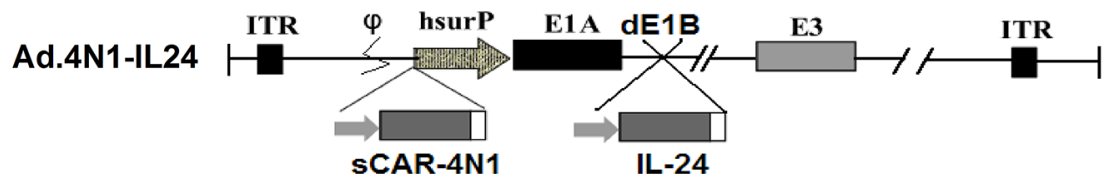

b
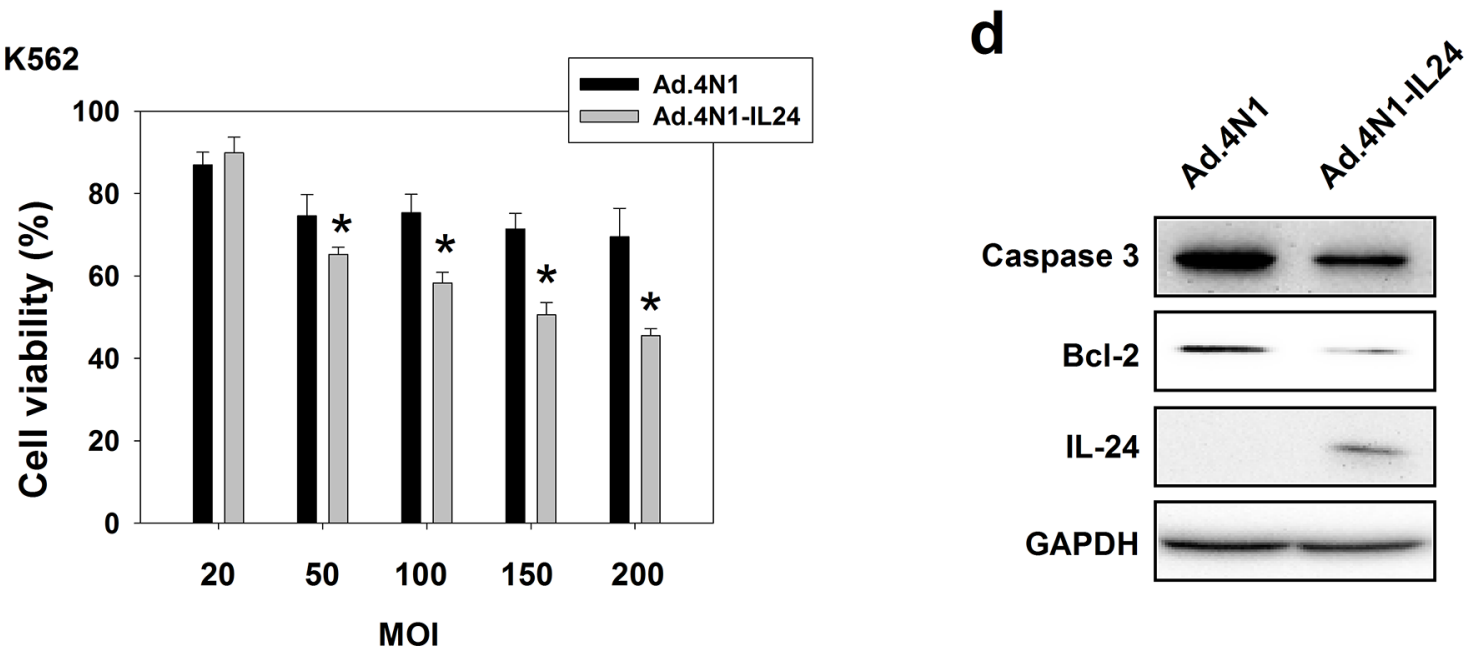

C

HL60

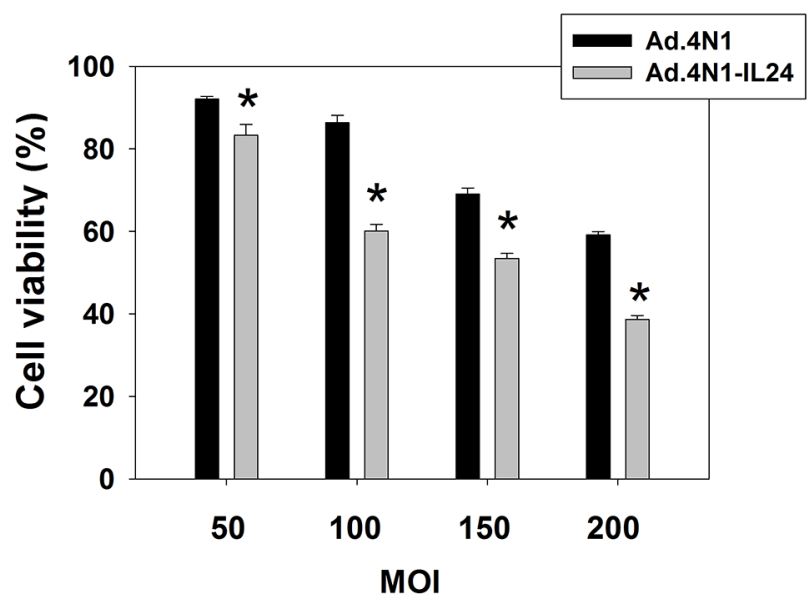

e

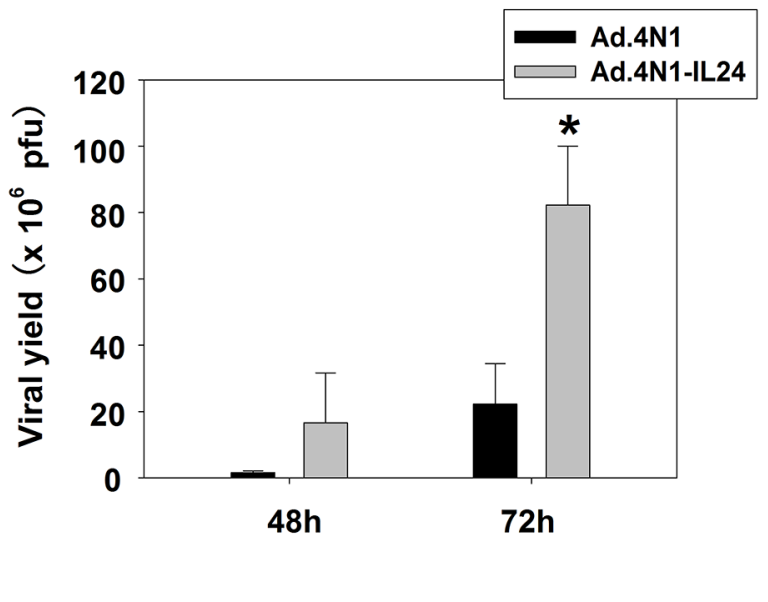

Figure 4: Ad.4N1-IL24 elicited higher cytotoxicity to leukemia cells in vitro. a. Schematic structure of Ad.4N1-IL24. Viral E1B was deleted (dE1B), and the resulted restriction site was used to harbor the gene encoding IL-24. b. K562 or c. HL60 cells were treated with Ad.4N1 or Ad.4N1-IL24 at MOIs indicated for $72 \mathrm{~h}$. Cell viability was analyzed by MTT assay. Values were calculated as percent of PBS control and presented as mean \pm SEM. $(* p<0.05)$ d. HL60 cells treated with Ad.4N1 or Ad.4N1-IL24 at 200 MOI for 48 h were analyzed by Western blot for levels of Caspase 3, Bcl-2, and IL-24. GAPDH served as the loading control. e. HL60 cells were treated with Ad.4N1 or Ad.4N1-IL24 at 100 MOI. After 48 h or 72 h, cells were collected and intracellular viruses were released by 3 freeze-thawing cycles. Viral production was determined through TCID50 assay. Values were shown as mean $\pm \operatorname{SEM} .\left({ }^{*} p<0.05\right)$ 
Ad.4N1-IL24 showed a dramatically lower level of cytotoxicity on BEASE-2B cells than on A549 cells, suggesting the safety of Ad.4N1-IL24. Furthermore, to investigate the underlying mechanism of Ad.4N1-IL24 induced higher cytotoxicity, HL60 cells infected with either Ad.4N1 or Ad.4N1-IL24 were investigated for the levels of caspase 3, Bcl-2, and IL-24. GAPDH served as the loading control. Our data showed that Ad.4N1-IL24 induced not only the expression of IL-24 in HL60 cells, but also more cleavage of caspase 3 as well as a downregulated level of survival factor Bcl-2 (Figure 4D). To determine viral replication capability, HL60 cells were infected with Ad.4N1 or Ad.4N1-IL24 and viral production after the time intervals was examined through TCID50 assay. Data showed that both Ad.4N1 and Ad.4N1-IL24 replicated in HL60 cells. Interestingly, Ad.4N1-IL24 replicated at a dramatically higher rate than Ad.4N1 (Figure 4E). Taken together, our data showed that Ad.4N1-IL24 not only altered apoptotic signaling pathway but also replicated much faster than Ad.4N1, which may be responsible for the higher cytotoxicity of Ad.4N1-IL24.

We then evaluated the in vivo antileukemia effect of oncolytic adenoviruses Ad.4N1 and Ad.4N1-IL24. HL60/Luc cells stably expressing fire fly luciferase were engrafted subcutaneously into NOD/SCID mice and monitored for bioluminescence. Mice were then grouped randomly and treated with Ad.4N1, Ad.4N1-IL24, or the control virus Ad.sp-E1A. The growth of HL60/ Luc xenograft posttreatment in individual mouse was evaluated by bioluminescence imaging in each group ( $n=$ 4) (Figure 5A-5C). Values for tumor size were shown in Supplementary Table S1. Data showed that the Ad.4N1IL24 treatment led to lower cancer cell burden than other treatments, and a significant difference was achieved between Ad.sp-E1A and Ad.4N1-IL24 groups at day 22 (Figure 5D). Apoptotic cells were detected in HL60/ Luc xenografts treated with Ad.4N1-IL24 (Figure 5E). Although Ad.4N1-IL24 did not prolong the survival of HL60/Luc xenograft mice as compared to control viruses (data not shown), our data have demonstrated the in vivo antileukemia effect of Ad.4N1-IL24 on HL60 leukemia xenografts through reducing cancer cell burden.

\section{DISCUSSION}

Based on our previous studies that oncolytic adenoviruses harboring sCAR-IL3 expression cassette successfully infected CD123+ leukemia cells, we proposed that engineering oncolytic adenoviruses to express sCARligand fusion proteins may provide a universal strategy to redirect oncolytic adenoviruses to various membrane receptors on cancer cells resisting to Ad5 infection. In the work presented, we demonstrated that Ad.4N1 successfully infected and suppressed in vitro proliferation of CD47+ K562 and HL60 leukemia cells. The in vitro infection and antiproliferative effect of Ad.4N1 was determined to be mediated by the 4N1-CD47 interaction. We further engineered Ad.4N1 to harbor a gene encoding IL-24, forming Ad.4N1-IL24, which successfully elicited in vivo antileukemia effect on a HL60 xenograft model. Although Ad.4N1 did not suppress in vivo leukemia growth as shown in Figure 5, our data have demonstrated that it could act as a useful tool for delivering anticancer genes into CD47+ leukemia cells. Therefore, our data indicated that oncolytic adenoviruses carrying gene encoding sCAR-4N1 fusion protein is a novel strategy to redirect oncolytic adenoviruses to CD47+ cancer cells.

TSP-1 has been extensively studied as an antitumor factor due to its antiangiogenic effect on papillary thyroid carcinoma [35], breast cancer [36], and melanoma [37]. In mammary tumor, TSP-1 was shown to inhibit angiogenesis, but promote tumor metastasis [38]. TSP1 secreted by glioma cells was also linked to promoting cell motility, suggesting an effect of TSP-1 in enhancing glioma metastasis [39]. CD36 has been recognized as the primary receptor for TSP-1 in inhibiting angiogenesis [40] as well as promoting metastasis [38], and the type I repeat domain of TSP-1 mediates the TSP-1-CD36 interaction. In addition to CD36, TSP-1 also acts as a ligand for $\mathrm{CD} 47$, and the C-terminal domain 4N1 peptide of TSP-1, is responsible for the TSP-1-CD47 binding [28, 41]. The 4N1 peptide has been shown to induce apoptosis or autophagy in various cancer cells, including chronic lymphocytic leukemia cells [42], breast cancer cells [43], and some Ras-expressing cancer cells [44]. However, 4N1 was also shown to inhibit apoptosis induced by camptothecin or doxorubicin in thyroid carcinoma cells [41]. In our results, sCAR-4N1 expressing oncolytic adenovirus Ad.4N1 elicited cytotoxicity to K562 and HL60 leukemia cells in vitro, but did not suppress the in vivo growth of HL60 cells. Our results and others have suggested diverse effects of the 4N1-CD47 interaction on different cancer cells under varied conditions. A previous study showed that the 4N1-CD47 interaction led to the binding of CD47 with $\alpha \mathrm{IIb} \beta 3$ integrin, which subsequently changed $\alpha \mathrm{IIb} \beta 3$ integrin into a high affinity state and promoted cell adhesion [45]. In fact, different from in vitro cultured leukemia cells, in vivo leukemia cells adhere to various extracellular matrix components and other cell types. Therefore, we propose here that cell adhesion status modulated by the 4N1-CD47 interaction could in turn become an important factor inhibiting the oncolytic adenoviruses as well as other therapeutic agents induced apoptosis signaling, pending further investigations.

Cytokine mda-7/IL-24 has been extensively investigated for selective antitumor effect on prostate cancer [46], lung cancer [47], ovarian cancer [48], breast carcinoma [49], colorectal cancer [50], and glioma [51]. Through gene delivery by a replicationdeficient adenovirus vector (Ad.mda-7/IL-24), the antitumor effect of IL-24 is achieved through both ectopic expression [47] and secretion [52]. IL-24 induces 
a
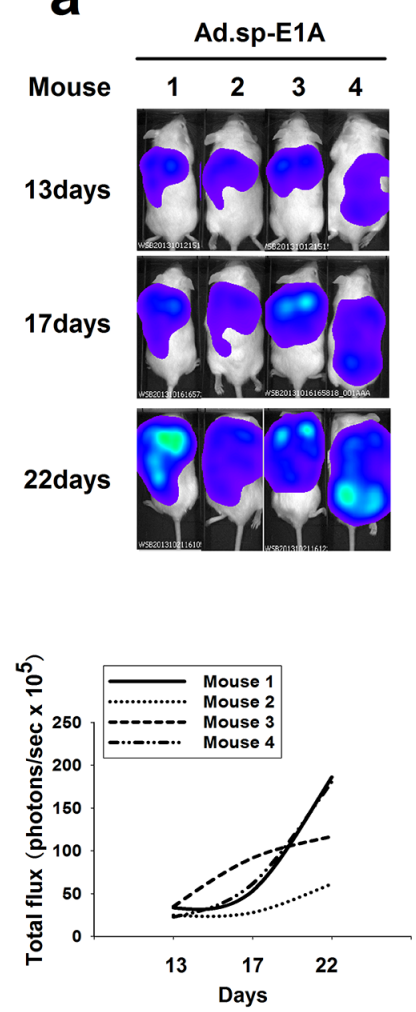

b

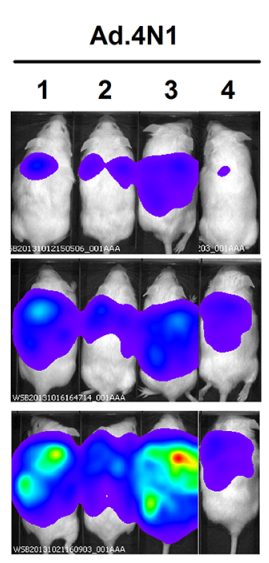

C
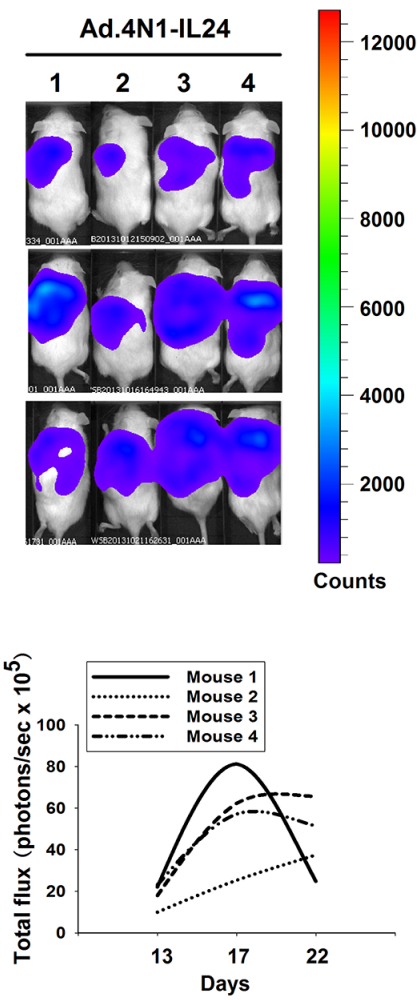

d

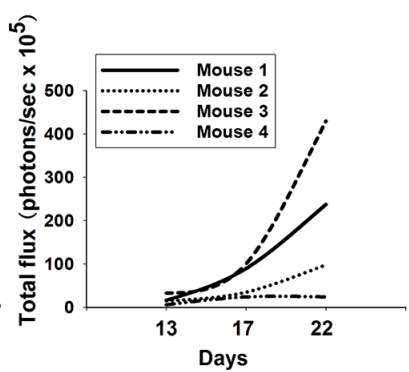

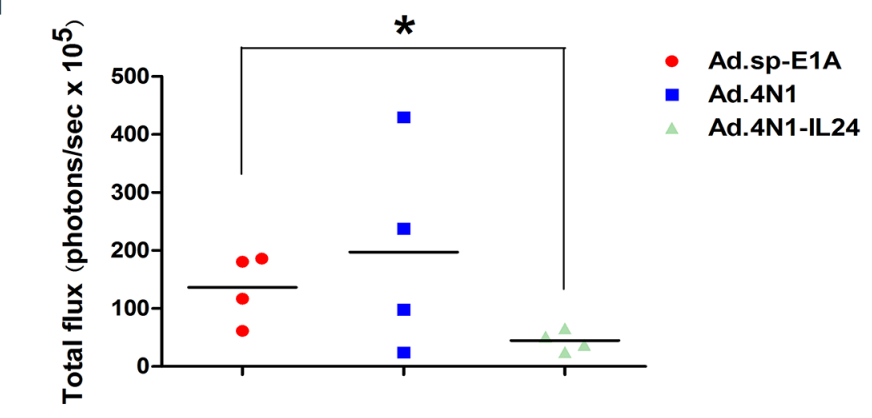

e
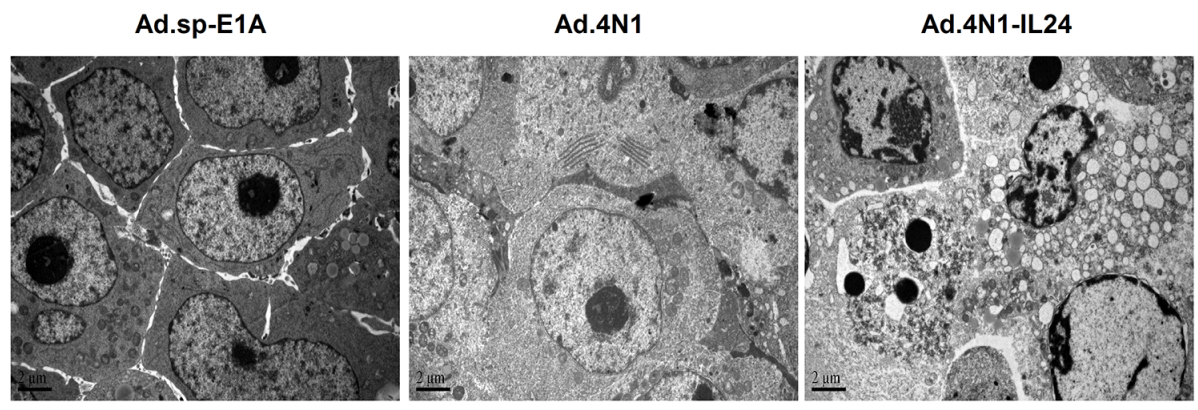

Figure 5: The in vivo antileukemia effect of Ad.4N1-IL24. The growth of HL60/Luc cells in mice treated with $2 \times 10^{9} \mathrm{pfu}$ of Ad.sp-E1 A a. Ad.4N1 b. or Ad.4N1-IL24 c. was evaluated by bioluminescence imaging individually at the day 13, 17, and 22 posttreatment $(n=4)$. The bioluminescence images of HL60/Luc engrafted mice from each group at the day 13, 17, and 22 posttreatment were shown on the top. Quantitative value of leukemia cell burden in individual mouse (photons $/ \mathrm{sec} \times 10^{5}$ ) was shown at the bottom. $\mathbf{d}$. The quantitative analysis of total leukemia cell burden in mice (photons/sec $\left.\times 10^{5}\right)$ at the day 22 posttreatment $\left({ }^{*} p<0.05\right.$ ). e. Apoptotic cells in HL60/Luc xenografts treated with Ad.4N1-IL24 were observed under TEM, as compared to Ad.sp-E1A and Ad.4N1 treatments. Bars show $2 \mu \mathrm{m}$. 
cancer selective apoptosis through interacting with endoplasmic reticulum (ER) chaperone protein $\mathrm{BiP} /$ GRP78 [53], activating Fas/FasL signaling pathway [48], as well as reduction in myeloid cell leukemia-1 (Mcl1) expression [54]. Ad.mda-7/IL-24 has entered clinical studies, and significant effectiveness and safety have been demonstrated [55]. However, some studies suggested that conditionally replicative oncolytic adenoviruses armed with IL-24 would be more effective [56, 57]. Although high level IL-24 was linked to promoting chronic lymphocytic leukemia cell survival through activating p38 MAPK [58], several other preclinical studies demonstrated that the gene delivery of IL-24 through oncolytic adenoviruses elicited significant antileukemia effect in vitro and in vivo $[19,59]$. In our in vitro and in vivo studies, the gene delivery of IL-24 through a CD47 targeting oncolytic adenovirus significantly enhanced antileukemia effect than the vector alone, which has not been shown in other studies. Furthermore, we demonstrated that the level of survival factor Bcl-2 upregulated by Ad.4N1 could be suppressed by the deletion of viral E1B and further harboring IL-24, and Ad.4N1-IL24 replicated at a dramatically higher rate than Ad.4N1, which may be responsible for the higher antiproliferative effect of Ad.4N1-IL24 on leukemia cells. Therefore, our results demonstrated that CD47-retargeted oncolytic adenoviruses armed with IL-24 could be valuable for the treatment of CD47+ leukemia.

In general, we provided a novel strategy to redirect oncolytic adenoviruses to CD47+ leukemia cells though carrying a sCAR-4N1 expression cassette in the viral genome, forming Ad.4N1. The in vitro infection and cytotoxicty of Ad.4N1 in leukemia cells was determined to be mediated by the 4N1-CD47 interaction. Furthermore, Ad.4N1 armed with the gene encoding IL-24 achieved significant antileukemia effects both in vitro and in vivo. Our data suggest that further clinical investigation into the antileukemia effect of Ad.4N1-IL24 may provide a novel antileukemia agent for future CD47+ leukemia therapies.

\section{MATERIALS AND METHODS}

\section{Cells}

Leukemia cell lines K562 and HL60 were obtained from American Type Culture Collection (ATCC, Rockville, MD, USA). Cells were routinely cultured in RPMI1640 (Hyclone Laboratories, Logan, UT, USA) supplemented with $10 \%$ fetal bovine serum (FBS, Hyclone Laboratories). The human embryonic kidney cell HEK293 was obtained from Microbix Biosystems (Toronto, ON, Canada) and cultured in Dulbecco's modified Eagle's medium (DMEM; GIBCOBRL, Grand Island, NY, USA) supplemented with $10 \%$ fetal bovine serum. All cells were kept at $37^{\circ} \mathrm{C}$ in a $95 \%$ air $5 \% \mathrm{CO}_{2}$ humidified incubator.

\section{Recombinant protein preparation}

To construct plasmid pOE30-sCAR-4N1, a twostep PCR was used to generate $s C A R-4 N 1$ gene from a previously reported plasmid pQE30-sCAR-IL3 [26]. The first PCR used pOE30-sCAR-IL3 as the template. The primers were 5'-CCCAAGCTTATGGCGCTCCTGCTGT GCTT-3' and 5'-TCACAACATAAAAGCGGGATCCAG GGGCG-3'. The second PCR used the product of the first PCR as the template. The primers were 5'-CCCAAGCTTATGGCGCTCCTGCTGTGCTT-3' and 5'-CCGAAGCTT TTAGGATCCAGGGGCG GAAG-3'. The product of the second PCR was inserted into the HindIII site of pQE30 to generate pOE30-sCAR-4N1. The his-tagged SCAR-4N1 fusion proteins were produced in Escherichia coli M15, extracted from inclusion bodies, and refolded by standard methods.

\section{Construction of recombinant adenoviruses}

The plasmid pAd.sp-E1A was constructed previously [27]. The sCAR-4N1 expression cassette was inserted into pAd.sp-E1A to generate pAd.sp-E1A-sCAR-4N1. The NheI-AleI fragments from pAd.sp-E1A-sCAR-4N1 were replaced into the NheI-AleI regions in plasmids pAd邓E1B-IL24, to generate pAd.sp-E1A-sCAR-4N1-邓E1BIL24. Plasmids pAd.sp-E1A-sCAR-4N1 or pAd.sp-E1AsCAR-4N1-区E1B-IL24 was then co-transfected with an adenovirus packing plasmid pBHGE3 (Microbix Biosystems, Toronto, Canada) into HEK293 cells to generate oncolytic adenoviruses Ad.4N1 and Ad.4N1-IL24 through homologous recombination. The recombinant adenoviruses were isolated through three rounds of plaque purification in HEK293 cells. Large-scale purification of adenoviruses was performed by ultracentrifugation with cesium chloride. The viral titers were determined by TCID50 (median tissue culture infective dose) assay in HEK293 cells.

\section{Cytotoxicity assay}

Leukemia cells were plated on 96-well plates at $2 \times 10^{4}$ per well. Cells in 6-7 replication wells were then infected with viruses at indicated MOIs. PBS was used as a control. The cell viability was determined by 3-(4,5-dimethylthiazol-2-yl)-2,5-diphenyltetrazolium bromide (MTT) assay.

For blocking assays, K562 cells were plated on 96well plates at $2 \times 10^{4}$ per well. Cells were then treated with viruses at the indicated MOIs in combination with recombinant human CD47 Fc chimera (R\&D systems, Minneapolis, MN, USA) at concentrations indicated. Cell viability was then determined by MTT assay after $96 \mathrm{~h}$.

\section{Western blotting analysis}

The cell extracts were subjected to SDS-PAGE and electroblotted onto nitrocellulose membranes. The 
membranes were then blocked with Tris-buffered saline and Tween 20 contaning $5 \%$ of bovine serum albumin at room temperature for $1 \mathrm{~h}$, followed by incubation with primary antibodies overnight at $4^{\circ} \mathrm{C}$. The membranes were then washed and incubated with secondary antibodies for $1 \mathrm{~h}$ at room temperature. After washing with Tris-buffered saline, the bands were detected under a Tanon 5500 chemiluminescence image system (Tanon Inc., Shanghai, China).

Rabbit anti-caspase 3, Bcl-2, Bax, and GAPDH antibodies were purchased from Cell Signaling Technology Inc. (Danvers, MA, USA). Goat antibody against IL-24 was purchased from R\&D systems Inc. (Minneapolis, MN, USA). The HRP conjugated goat anti-rabbit and donkey anti-goat antibodies were purchased from MultiSciences (Lianke) Biotech Co., Ltd. (Hangzhou, China).

\section{Animal experiments}

Male NOD/SCID mice at 4-5 weeks of age were used for leukemia xenograft. HL-60/Luc cells at $6 \times 10^{6}$ cells/mouse were injected subcutaneously into the mice on the back. When leukemia burdens reached about 1 $\times 10^{5}$ photons/sec, mice were randomly grouped and in situ injected with $2 \times 10^{9}$ plaque-forming units (pfu) of oncolytic adenoviruses. Mice were injected with D-Luciferin, and bioluminescence was recorded under a Caliber IVIS kinetics (Caliper life sciences, USA). Regions of interest were assigned through the IVIS software and reported as area flux (photons/sec), defined by radiance (photons $/ \mathrm{sec} / \mathrm{cm}^{2} /$ steradian).

\section{Ethnic statement}

All animal studies were approved by the Institutional Animal Care and Use Committee (IACUC) of Zhejiang Chinese Medical University, Zhejiang, China.

\section{Statistical analysis}

Differences among the treatment groups were assessed by student's $t$-test. $P<0.05$ was considered significant.

\section{ACKNOWLEDGMENTS}

This work was supported by National Natural Science Foundation of China grants 30801379 and 81572986, Zhejiang Provincial Natural Science Foundation grant LZ16D060002, and Zhejiang Provincial Top Key Discipline of Biology.

\section{CONFLICTS OF INTEREST}

The authors declare no conflict of interest.

\section{REFERENCES}

1. Brown EJ, Frazier WA. Integrin-associated protein (CD47) and its ligands. Trends Cell Biol. 2001; 11:130-135.

2. Jaiswal S, Jamieson CH, Pang WW, Park CY, Chao MP, Majeti R, Traver D, van Rooijen N, Weissman IL. CD47 is upregulated on circulating hematopoietic stem cells and leukemia cells to avoid phagocytosis. Cell. 2009; 138:271-285.

3. Cioffi M, Trabulo S, Hidalgo M, Costello E, Greenhalf W, Erkan M, Kleeff J, Sainz B, Jr, Heeschen C. Inhibition of CD47 Effectively Targets Pancreatic Cancer Stem Cells via Dual Mechanisms. Clin Cancer Res. 2015.

4. Lee TK, Cheung VC, Lu P, Lau EY, Ma S, Tang KH, Tong M, Lo J, Ng IO. Blockade of CD47-mediated cathepsin S/protease-activated receptor 2 signaling provides a therapeutic target for hepatocellular carcinoma. Hepatology. 2014; 60:179-191.

5. Theocharides AP, Jin L, Cheng PY, Prasolava TK, Malko AV, Ho JM, Poeppl AG, van Rooijen N, Minden MD, Danska JS, Dick JE, Wang JC. Disruption of SIRPalpha signaling in macrophages eliminates human acute myeloid leukemia stem cells in xenografts. J Exp Med. 2012; 209:1883-1899.

6. Majeti R, Chao MP, Alizadeh AA, Pang WW, Jaiswal S, Gibbs KD, Jr., van Rooijen N, Weissman IL. CD47 is an adverse prognostic factor and therapeutic antibody target on human acute myeloid leukemia stem cells. Cell. 2009; 138:286-299.

7. Chao MP, Alizadeh AA, Tang C, Jan M, WeissmanTsukamoto R, Zhao F, Park CY, Weissman IL, Majeti R. Therapeutic antibody targeting of CD47 eliminates human acute lymphoblastic leukemia. Cancer Res. 2011; 71:1374-1384.

8. Willingham SB, Volkmer JP, Gentles AJ, Sahoo D, Dalerba P, Mitra SS, Wang J, Contreras-Trujillo H, Martin R, Cohen JD, Lovelace P, Scheeren FA, Chao MP, Weiskopf K, Tang C, Volkmer AK, et al. The CD47-signal regulatory protein alpha (SIRPa) interaction is a therapeutic target for human solid tumors. Proc Natl Acad Sci U S A. 2012; 109:6662-6667.

9. Weiskopf K, Ring AM, Ho CC, Volkmer JP, Levin AM, Volkmer AK, Ozkan E, Fernhoff NB, van de Rijn M, Weissman IL, Garcia KC. Engineered SIRPalpha variants as immunotherapeutic adjuvants to anticancer antibodies. Science. 2013; 341:88-91.

10. Wang Y, Xu Z, Guo S, Zhang L, Sharma A, Robertson GP, Huang L. Intravenous delivery of siRNA targeting CD47 effectively inhibits melanoma tumor growth and lung metastasis. Mol Ther. 2013; 21:1919-1929.

11. Tseng D, Volkmer JP, Willingham SB, Contreras-Trujillo H, Fathman JW, Fernhoff NB, Seita J, Inlay MA, Weiskopf K, Miyanishi M, Weissman IL. Anti-CD47 antibody-mediated phagocytosis of cancer by macrophages primes an effective antitumor T-cell response. Proc Natl Acad Sci U S A. 2013; 110:11103-11108. 
12. Liu XY. Targeting gene-virotherapy of cancer and its prosperity. Cell Res. 2006; 16:879-886.

13. Curiel DT. The development of conditionally replicative adenoviruses for cancer therapy. Clin Cancer Res. 2000; 6:3395-3399.

14. Hawkins LK, Lemoine NR, Kirn D. Oncolytic biotherapy: a novel therapeutic plafform. Lancet Oncol. 2002; 3:17-26.

15. Kirn D, Martuza RL, Zwiebel J. Replication-selective virotherapy for cancer: Biological principles, risk management and future directions. Nat Med. 2001; 7:781-787.

16. Russell SJ, Peng KW, Bell JC. Oncolytic virotherapy. Nat Biotechnol. 2012; 30:658-670.

17. Bewley MC, Springer K, Zhang YB, Freimuth P, Flanagan JM. Structural analysis of the mechanism of adenovirus binding to its human cellular receptor, CAR. Science. 1999; 286:1579-1583.

18. Bergelson JM, Cunningham JA, Droguett G, Kurt-Jones EA, Krithivas A, Hong JS, Horwitz MS, Crowell RL, Finberg RW. Isolation of a common receptor for Coxsackie B viruses and adenoviruses 2 and 5. Science. 1997; 275:1320-1323.

19. Qian W, Liu J, Tong Y, Yan S, Yang C, Yang M, Liu X. Enhanced antitumor activity by a selective conditionally replicating adenovirus combining with MDA-7/interleukin-24 for B-lymphoblastic leukemia via induction of apoptosis. Leukemia. 2008; 22:361-369.

20. Yotnda P, Zompeta C, Heslop HE, Andreeff M, Brenner MK, Marini F. Comparison of the efficiency of transduction of leukemic cells by fiber-modified adenoviruses. Hum Gene Ther. 2004; 15:1229-1242.

21. Nilsson M, Ljungberg J, Richter J, Kiefer T, Magnusson M, Lieber A, Widegren B, Karlsson S, Fan X. Development of an adenoviral vector system with adenovirus serotype 35 tropism; efficient transient gene transfer into primary malignant hematopoietic cells. J Gene Med. 2004; 6:631-641.

22. Saudemont A, Corm S, Wickham T, Hetuin D, Quesnel B. Induction of leukemia-specific CD8+ cytotoxic T cells with autologous myeloid leukemic cells maturated with a fibermodified adenovirus encoding TNF-alpha. Mol Ther. 2005; 11:950-959.

23. Grellier E, Lecolle K, Rogee S, Couturier C, D'Halluin JC, Hong SS, Fender P, Boulanger P, Quesnel B, Colin M. A fiber-modified adenoviral vector interacts with immunoevasion molecules of the B7 family at the surface of murine leukemia cells derived from dormant tumors. Mol Cancer. 2011; 10:105.

24. Itoh A, Okada T, Mizuguchi H, Hayakawa T, Mizukami H, Kume A, Takatoku M, Komatsu N, Hanazono Y, Ozawa $\mathrm{K}$. A soluble CAR-SCF fusion protein improves adenoviral vector-mediated gene transferto c-Kit-positive hematopoietic cells. The Journal of Gene Medicine. 2003; 5:929-940.

25. Li GC, Li N, Zhang YH, Li X, Wang YG, Liu XY, Qian WB, Liu XC. Mannose-exposing myeloid leukemia cells detected by the sCAR-PPA fusion protein. Int J Hematol. 2009; 89:611-617.

26. Li G, Li X, Wu H, Yang X, Zhang Y, Chen L, Wu X, Cui L, Wu L, Luo J, Liu XY. CD123 targeting oncolytic adenoviruses suppress acute myeloid leukemia cell proliferation in vitro and in vivo. Blood Cancer J. 2014; 4:e194.

27. Zhang KJ, Wang YG, Cao X, Zhong SY, Wei RC, Wu YM, Yue XT, Li GC, Liu XY. Potent antitumor effect of interleukin-24 gene in the survivin promoter and retinoblastoma double-regulated oncolytic adenovirus. Hum Gene Ther. 2009; 20:818-830.

28. Gao AG, Lindberg FP, Finn MB, Blystone SD, Brown EJ, Frazier WA. Integrin-associated protein is a receptor for the C-terminal domain of thrombospondin. J Biol Chem. 1996; 271:21-24.

29. Dash R, Bhoopathi P, Das SK, Sarkar S, Emdad L, Dasgupta S, Sarkar D, Fisher PB. Novel mechanism of MDA-7/IL-24 cancer-specific apoptosis through SARI induction. Cancer Res. 2014; 74:563-574.

30. Tian H, Wang J, Zhang B, Di J, Chen F, Li H, Li L, Pei D, Zheng J. MDA-7/IL-24 induces Bcl-2 denitrosylation and ubiquitin-degradation involved in cancer cell apoptosis. PLoS ONE. 2012; 7:e37200.

31. Wang Z, Sampath J, Fukuda S, Pelus LM. Disruption of the inhibitor of apoptosis protein survivin sensitizes Bcrabl-positive cells to STI571-induced apoptosis. Cancer Res. 2005; 65:8224-8232.

32. Abitorabi MA, Pachynski RK, Ferrando RE, Tidswell M, Erle DJ. Presentation of integrins on leukocyte microvilli: a role for the extracellular domain in determining membrane localization. J Cell Biol. 1997; 139:563-571.

33. Carter BZ, Wang RY, Schober WD, Milella M, Chism D, Andreeff M. Targeting Survivin expression induces cell proliferation defect and subsequent cell death involving mitochondrial pathway in myeloid leukemic cells. Cell Cycle. 2003; 2:488-493.

34. Stachelek SJ, Finley MJ, Alferiev IS, Wang F, Tsai RK, Eckells EC, Tomczyk N, Connolly JM, Discher DE, Eckmann DM, Levy RJ. The effect of CD47 modified polymer surfaces on inflammatory cell attachment and activation. Biomaterials. 2011; 32:4317-4326.

35. Tanaka K, Sonoo H, Kurebayashi J, Nomura T, Ohkubo S, Yamamoto Y, Yamamoto S. Inhibition of infiltration and angiogenesis by thrombospondin-1 in papillary thyroid carcinoma. Clin Cancer Res. 2002; 8:1125-1131.

36. Zhang YW, Su Y, Volpert OV, Vande Woude GF. Hepatocyte growth factor/scatter factor mediates angiogenesis through positive VEGF and negative thrombospondin 1 regulation. Proc Natl Acad Sci U S A. 2003; 100:12718-12723.

37. Sund M, Hamano Y, Sugimoto H, Sudhakar A, Soubasakos M, Yerramalla U, Benjamin LE, Lawler J, Kieran M, Shah A, Kalluri R. Function of endogenous inhibitors of 
angiogenesis as endothelium-specific tumor suppressors. Proc Natl Acad Sci U S A. 2005; 102:2934-2939.

38. Yee KO, Connolly CM, Duquette M, Kazerounian S, Washington R, Lawler J. The effect of thrombospondin-1 on breast cancer metastasis. Breast Cancer Res Treat. 2009; 114:85-96.

39. Amagasaki K, Sasaki A, Kato G, Maeda S, Nukui H, Naganuma H. Antisense-mediated reduction in thrombospondin-1 expression reduces cell motility in malignant glioma cells. Int J Cancer. 2001; 94:508-512.

40. Simantov R, Febbraio M, Crombie R, Asch AS, Nachman RL, Silverstein RL. Histidine-rich glycoprotein inhibits the antiangiogenic effect of thrombospondin-1. J Clin Invest. $2001 ; 107: 45-52$.

41. Rath GM, Schneider C, Dedieu S, Rothhut B, SoulaRothhut M, Ghoneim C, Sid B, Morjani H, El Btaouri H, Martiny L. The C-terminal CD47/IAP-binding domain of thrombospondin-1 prevents camptothecin- and doxorubicin-induced apoptosis in human thyroid carcinoma cells. Biochim Biophys Acta. 2006; 1763:1125-1134.

42. Mateo V, Lagneaux L, Bron D, Biron G, Armant M, Delespesse G, Sarfati M. CD47 ligation induces caspaseindependent cell death in chronic lymphocytic leukemia. Nat Med. 1999; 5:1277-1284.

43. Manna PP, Frazier WA. CD47 mediates killing of breast tumor cells via Gi-dependent inhibition of protein kinase A. Cancer Res. 2004; 64:1026-1036.

44. Kalas W, Swiderek E, Switalska M, Wietrzyk J, Rak J, Strzadala L. Thrombospondin-1 receptor mediates autophagy of RAS-expressing cancer cells and triggers tumour growth inhibition. Anticancer Res. 2013; 33:1429-1438.

45. Fujimoto TT, Katsutani S, Shimomura T, Fujimura K. Thrombospondin-bound integrin-associated protein (CD47) physically and functionally modifies integrin alphaIIbbeta3 by its extracellular domain. J Biol Chem. 2003; 278:26655-26665.

46. Lebedeva IV, Su ZZ, Sarkar D, Kitada S, Dent P, Waxman $\mathrm{S}$, Reed JC, Fisher PB. Melanoma differentiation associated gene-7, mda-7/interleukin-24, induces apoptosis in prostate cancer cells by promoting mitochondrial dysfunction and inducing reactive oxygen species. Cancer Res. 2003; 63:8138-8144.

47. Ramesh R, Ito I, Gopalan B, Saito Y, Mhashilkar AM, Chada S. Ectopic production of MDA-7/IL-24 inhibits invasion and migration of human lung cancer cells. Mol Ther. 2004; 9:510-518.

48. Gopalan B, Litvak A, Sharma S, Mhashilkar AM, Chada S, Ramesh R. Activation of the Fas-FasL signaling pathway by MDA-7/IL-24 kills human ovarian cancer cells. Cancer Res. 2005; 65:3017-3024.

49. Sarkar D, Su ZZ, Vozhilla N, Park ES, Gupta P, Fisher PB. Dual cancer-specific targeting strategy cures primary and distant breast carcinomas in nude mice. Proc Natl Acad Sci U S A. 2005; 102:14034-14039.

50. Emdad L, Lebedeva IV, Su ZZ, Sarkar D, Dent P, Curiel DT, Fisher PB. Melanoma differentiation associated gene-7/interleukin-24 reverses multidrug resistance in human colorectal cancer cells. Mol Cancer Ther. 2007; 6:2985-2994.

51. Park MA, Yacoub A, Sarkar D, Emdad L, Rahmani M, Spiegel S, Koumenis C, Graf M, Curiel DT, Grant S, Fisher PB, Dent P. PERK-dependent regulation of MDA-7/ IL-24-induced autophagy in primary human glioma cells. Autophagy. 2008; 4:513-515.

52. Su Z, Emdad L, Sauane M, Lebedeva IV, Sarkar D, Gupta P, James CD, Randolph A, Valerie K, Walter MR, Dent $\mathrm{P}$, Fisher PB. Unique aspects of mda-7/IL-24 antitumor bystander activity: establishing a role for secretion of MDA-7/IL-24 protein by normal cells. Oncogene. 2005; 24:7552-7566.

53. Gupta P, Walter MR, Su ZZ, Lebedeva IV, Emdad L, Randolph A, Valerie K, Sarkar D, Fisher PB. BiP/GRP78 is an intracellular target for MDA-7/IL-24 induction of cancer-specific apoptosis. Cancer Res. 2006; 66:8182-8191.

54. Dash R, Richards JE, Su ZZ, Bhutia SK, Azab B, Rahmani M, Dasmahapatra G, Yacoub A, Dent P, Dmitriev IP, Curiel DT, Grant S, Pellecchia M, Reed JC, Sarkar D, Fisher PB. Mechanism by which Mcl-1 regulates cancer-specific apoptosis triggered by mda-7/IL-24, an IL-10-related cytokine. Cancer Res. 2010; 70:5034-5045.

55. Tong AW, Nemunaitis J, Su D, Zhang Y, Cunningham C, Senzer N, Netto G, Rich D, Mhashilkar A, Parker K, Coffee K, Ramesh R, Ekmekcioglu S, Grimm EA, van Wart Hood $\mathrm{J}$, Merritt J, et al. Intratumoral injection of INGN 241, a nonreplicating adenovector expressing the melanomadifferentiation associated gene-7 (mda-7/IL24): biologic outcome in advanced cancer patients. Mol Ther. 2005; 11:160-172.

56. Jiang G, Liu YQ, Wei ZP, Pei DS, Mao LJ, Zheng JN. Enhanced anti-tumor activity by the combination of a conditionally replicating adenovirus mediated interleukin-24 and dacarbazine against melanoma cells via induction of apoptosis. Cancer Lett. 2010; 294:220-228.

57. Liu XY, Gu JF. Targeting gene-virotherapy of cancer. Cell Res. 2006; 16:25-30.

58. Sainz-Perez A, Gary-Gouy H, Portier A, Davi F, MerleBeral H, Galanaud P, Dalloul A. High Mda-7 expression promotes malignant cell survival and p38 MAP kinase activation in chronic lymphocytic leukemia. Leukemia. 2006; 20:498-504.

59. Wei X, Liu L, Wang G, Li W, Xu K, Qi H, Liu H, Shen J, Li Z, Shao J. Potent antitumor activity of the Ad5/11 chimeric oncolytic adenovirus combined with interleukin-24 for acute myeloid leukemia via induction of apoptosis. Oncol Rep. 2015; 33:111-118. 